\title{
Quantum Evolutionary Algorithm for Chemical Parallel Flow Shop Scheduling Problem
}

\author{
Qi Tang ${ }^{1, a}$, Peng Liu ${ }^{1}$ \\ ${ }^{1}$ Management School, \\ Shenyang University of Technology, \\ Shenyang, China, 110870 \\ atangqi20050708@163.com
}

\author{
Jianxun Tang ${ }^{2}$ \\ ${ }^{2}$ College of Air Traffic Management, \\ Civil Aviation University of China, \\ Tianjin, China, 300300
}

\author{
Xiang $\mathrm{Li}^{1}$ \\ ${ }^{1}$ Management School, \\ Shenyang University of Technology, \\ Shenyang, China, 110870
}

\begin{abstract}
This paper studies the typical problem of integration of chemical batching and scheduling, which is characterized by multi-product, multi-stage, parallel production, production coordination between stages, limit of equipment production capacity and inventory capacity, parallel equipment selection and limitation of batch quantity. The optimization objective of this problem is to minimize the make-span by arranging batch number and size, allocating machines, making decision about the processing sequence and scheduling time of batch on a machine on the premise that the equipment production capacity, maximum inventory and material supply coordination are met. This paper presents modified quantum evolutionary algorithm(MQEA). The computational results show that the MQAE may find optimal or suboptimal solutions in a short run time for all the instances.
\end{abstract}

Keywords-chemical industry; quantum evolutionary algorithm; batch scheduling

\section{INTRODUCTION}

Integration of batching and scheduling in chemical industries has been the subject of extensive research since the early 1990s. Comparing with separate batching and scheduling, the integration can shorten the production time. The main focus is on the appropriate decision of batch size on units and efficient allocation of one or more resources to batches over time. There are limited literatures on the integration problem of batching and scheduling. As far as the production environment is concerned, integration of batching and scheduling in chemical industries can be classified into two main groups such as singe stage and multiple stages. As for single-stage, Erdirik-Dogan and Grossmann (2007) ${ }^{[1]}$ present a multi-period mixed integer linear programming (MILP) formulation to deal with parallel units, which is effective for small size. They propose bi-level composition algorithm to decompose the problem into batching and scheduling sub-problems. The sub-problems were resulted corporately. Liu and Karimi (2005) ${ }^{[2]}$ present a MILP formulation which can deal with hybrid FS using a continuous-time representation with synchronous slots and a new idea of several balances such as time, mass, resource.

In the previous literature, most are based on constructed heuristics and MILP models ${ }^{[3-11]}$. Constructed heuristics use shorter time to resolve a problem. However, the quality of solutions could not be satisfied. The construction of the heuristics is designed according to optimal characteristics and other concrete features, which cause worse commonality and optimality. There are some MILP modeling ideas in the literatures of chemical industries which can be considered for reference to deal with a general problem on FS.

A method of solving the problem of chemical batch scheduling based on Quantum Evolutionary Algorithm is brought forward in this paper. This paper analyzes the characteristics of the batch scheduling problem in the first place and put forward some preliminary nature to reduce the scale of problem before solving problem through particle swarm optimization. Particle coding method is designed on the basis of the characteristics of the batch scheduling problem and the particle position vector is encoded into scheduling plan. In order to deal with the related constraints in the batch scheduling problem, a new constraints handling mechanism is introduced into the Quantum Evolutionary Algorithm, which includes batch separation strategy and scheduling generation method.

\section{PROBLEM DESCRIPTION}

Integration of batching and scheduling in a parallel flow shop is to make decision about the total production quantity, batch number, size and schedule of each device at each stage on the condition that the demand for each product is known in a multi-stage production environment where there is at least one stage having parallel device and 
multiple products can be produced. We analyze some character of the integration problem as follow:

Definition 1 The difference between starting time of latter job and ending time of former job is called span from ending to beginning. The ending time is represented by $C_{i}$. In case of adjacent relationship from $i$ to $j$, the span from ending to beginning of $i$ and $j$ is $S_{j}-C_{i}$.

Definition 2 If the time span is not less than a certain number, this number is called the minimum time span, which is represented by $d_{i, j}^{\text {min }}$ shown as Figure 1. Unless otherwise specified, $d_{i, j}^{\mathrm{min}}$ is interpreted as the time span from beginning to beginning $S_{j}-S_{i} \geq d_{i, j}^{\min }$ during study.

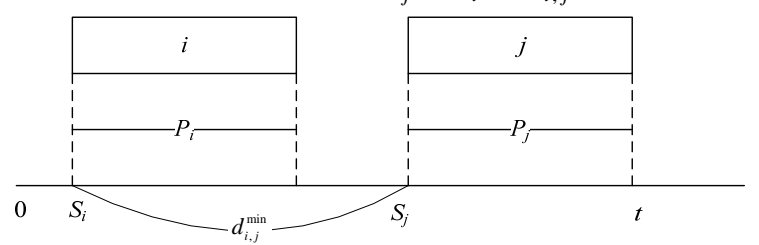

Figure 1. minimum time span

Certain value of $d_{i, j}^{\min }$ indicates certain relationship between different jobs. If $d_{i, j}^{\min }=p_{j}$, it indicates that job $j$ can start after completion of job $i$. If $d_{i, j}^{\min }<p_{j}$, it indicates that job $j$ and $i$ can be processed in parallel and the starting time is after $S_{i}+d_{i, j}^{\min }$. If $d_{i, j}^{\min }=0$, it indicates that $j$ and $i$ can be processed in parallel, but $j$ starts after $i$.

Definition 3 One of the real jobs in the project which can start in the first place is called initial job.

Definition 4 One of the real jobs in the project which can start at last is called terminal job.

\section{MODIFIED QUANTUM EVOLUTIONARY ALGORITHM}

The main feature of modified quantum evolutionary algorithm is to modify the location updating method of particle swarm optimization by combination with the theory of quantum physics. The current local and global optimal location information of each particle shall be taken into consideration when updating the particle position.

In order to solve the batch scheduling more effectively, we improve the current particle population and generate individual solution of better quality so as to update the global and individual optimal position by adoption of quantum behavior during the optimum iterative procedure of the algorithm. Mixed algorithm realizes the collaborative optimization process and guarantees the evolutionary process of particle population towards the solution of better quality efficiently and orderly. The complete steps of the algorithm are as follows:
Step1. Set the parameters of particle and give the relevant parameters of modified quantum evolutionary algorithm.

Step2. Set the position and speed range of each particle.

Step3. Generate initial population at random within the position and speed range of the particles.

Step4. Decode the particles into scheduling time of batch in equipment according to the preliminary characteristics, and then calculate the objective function value.

Step5. Evaluate the objective function value of the particle individual in the population.

Step6. Update both the individual optimal record of the particles in the population and the global optimal record of the whole population.

Step7. Select two particles at random in the population, get their individual position vectors, and then get the difference vector.

Step8. Select another particle, and generate variation vector through interaction of its position vector with the difference vector.

Step9. Select the individual optimal solution of the particles at random, cross it with the variation vector, and then get the reference vector.

Step10. Compare the individual optimal solution with the reference vector.

Step11. If the reference vector is better than the individual optimal solution, replace the individual optimal solution. Otherwise, turn to Step 14.

Step12. Compare the reference vector with the global optimal solution.

Step13. If the reference vector is better than the global optimal solution, replace the global optimal solution.

Step14. Determine whether the termination criterion is met or not. If yes, turn to Step 16 .

Step15. Update the speed and position of the particles in the population. Repeat Step 4 to 15.

Step16. The algorithm terminates. Output the optimal record of the global optimal particles.

\section{EXPERIMENTAL RESULTS}

The demand for each group of products generated from set of examples at random is set as $[2000+100 h, 2000+100(h+1)] \forall h \in z, 50 \leq h<80$

. The difference between example demands next to each other is 100 . According to the data shown in Table 1 , the parameters generated at random, including production batch size, maximum limit of inventory between stages, fixed and variable coefficients of batch processing time, obey uniform distribution. Their distribution ranges are $[150,200],[200,250],[0.5,1.5]$ and $[0.002,0.02]$. The set of test examples is generated by computer at random. Table I shows 20 groups of test results. Column "Cplex", "PSO" and "MQEA" are the best objective function values obtained respectively by software computation, standard particle swarm optimization algorithm and modified quantum evolutionary algorithm. "CPUc" shows the best 
earliest time obtained by Cplex software computation. "CPUp" and "CPUm" show the computing times of the standard particle swarm optimization and the modified quantum evolutionary algorithm respectively. The unit of time is second.

TABLE I. COMPUTATIONAL RESULTS

\begin{tabular}{|c|c|c|c|c|c|c|c|}
\hline instance & demand & Cplex & PSO & MQEA & CPUc(s) & CPUp(s) & CPUm(s) \\
\hline 1 & 7000 & 1.203 & 1.139 & 1.0000 & 12.080 & 0.001 & 0.257 \\
\hline 2 & 7100 & 1.199 & 1.154 & 1.0000 & 15.088 & 0.001 & 0.567 \\
\hline 3 & 7200 & 1.176 & 1.142 & 1.0000 & 18.097 & 0.001 & 0.478 \\
\hline 4 & 7300 & 1.173 & 1.146 & 1.0000 & 20.280 & 0.001 & 0.678 \\
\hline 5 & 7400 & 1.203 & 1.134 & 1.0000 & 19.580 & 0.002 & 0.789 \\
\hline 6 & 7500 & 1.234 & 1.130 & 1.0000 & 17.547 & 0.002 & 0.689 \\
\hline 7 & 7600 & 1.211 & 1.157 & 1.0000 & 13.654 & 0.002 & 0.765 \\
\hline 8 & 7700 & 1.215 & 1.170 & 1.0000 & 18.864 & 0.002 & 0.890 \\
\hline 9 & 7800 & 1.219 & 1.169 & 1.0000 & 19.921 & 0.001 & 0.876 \\
\hline 10 & 7900 & 1.194 & 1.173 & 1.0000 & 20.587 & 0.002 & 0.908 \\
\hline 11 & 8000 & 1.195 & 1.193 & 1.0000 & 24.987 & 0.002 & 0.976 \\
\hline 12 & 8100 & 1.189 & 1.151 & 1.0000 & 24.123 & 0.002 & 0.997 \\
\hline 13 & 8200 & 1.191 & 1.152 & 1.0000 & 28.347 & 0.001 & 1.123 \\
\hline 14 & 8300 & 1.199 & 1.148 & 1.0000 & 31.254 & 0.001 & 1.231 \\
\hline 15 & 8400 & 1.178 & 1.140 & 1.0000 & 31.365 & 0.001 & 1.439 \\
\hline 16 & 8500 & 1.183 & 1.135 & 1.0000 & 32.388 & 0.002 & 1.525 \\
\hline 17 & 8600 & 1.207 & 1.137 & 1.0000 & 35.598 & 0.001 & 1.642 \\
\hline 18 & 8700 & 1.237 & 1.148 & 1.0000 & 45.647 & 0.001 & 1.774 \\
\hline 19 & 8800 & 1.247 & 1.165 & 1.0000 & 34.987 & 0.001 & 1.876 \\
\hline 20 & 8900 & 1.211 & 1.200 & 1.0000 & 35.654 & 0.001 & 1.879 \\
\hline
\end{tabular}

\section{CONCLUSION}

The problem of integration of batching and scheduling in hybrid flow shop has attracted broad attention from many scholars in the academic world and become the research focus of production scheduling problem in petrochemical industry. The problem requires two aspects of decision-making, batching and scheduling. Batching means to decide appropriate number of batches and batch size for each process of each product. Scheduling means to arrange starting time of each batch. This paper analyze the characters depending on batch direct relation and proposes modified quantum evolutionary algorithm after getting priority relationship among batches under the premise that the material supply and inventory constraint are satisfied. The experimental results verify the accuracy and efficiency of the modified quantum evolutionary algorithm and confirm that the algorithm put forward in this paper is an effective method to solve the problem of integration of batching and scheduling in parallel flow shop.

\section{ACKNOWLEDGMENT}

This work is supported by National Natural Science Foundation of China under Grant (71001074); Humanity Social Science Youth foundation of Ministry of Education of China under Grant (13YJC630146); Soft Science Project of Shenyang Science and Technology Bureau ( F13-315-5-29). 


\section{REFERENCES}

[1] M. Erdirik, I.E. Grossmann, J. Wassick: 17th European Symposium on Computer Aided Process Engineering Vol. 24(2007), p. 625-630

[2] A. Sundaramoorthy, I.A. Karimi: Chemical Engineering Science Vol. 60(10)(2005), p. 2679- 2702.

[3] K. Neumann, C. Schwindt, N. Trautmann: OR Spectrum Vol. 24(2002), p. 251-279.

[4] M.A. Shaik, C.A. Floudas: Computers and Chemical Engineering Vol. 32(2008), p. 260-274.

[5] P.M. Castro, I.E. Grossmann: Industrial and Engineering Chemistry Research Vol. 44(24)(2005), p. 9175-9190.
[6] Y. Liu, I.A. Karimi: Chemical Engineering Science Vol. 62(2007), p. 1549-1566.

[7] C.A. Floudas, X.L. Lin: Computer and Chemical Engineering Vol. 28(11)(2004), p. 2109-2114.

[8] L. Liu, C. Ng, T.Cheng: Vol. 8(1)(2014), p. 307-318.

[9] S. Bose, S. Bhattacharya: Computers and Chemical Engineering Vol. 33(1)(2009), p. 287-295.

[10] B. Shi, L. Yan, W.Wu: Industrial and Engineering Chemistry Research Vol. 51(2012), p. 8535- 8549.

[11] E. Ghafari, R.Sahraeian: International Journal of Industrial Engineering and Production Research Vol. 25(1)(2014), p. 55-63. 